\title{
Photoelectron spectroscopy and modeling of interface properties related to organic photovoltaic cells
}

Mats Fahlman, Parisa Sehati, Wojciech Osikowicz, Slawomir Braun, Michel P. de Jong and Geert Brocks

\section{Linköping University Post Print}

\section{Tweet}

N.B.: When citing this work, cite the original article.

Original Publication:

Mats Fahlman, Parisa Sehati, Wojciech Osikowicz, Slawomir Braun, Michel P. de Jong and Geert Brocks, Photoelectron spectroscopy and modeling of interface properties related to organic photovoltaic cells, 2013, Journal of Electron Spectroscopy and Related Phenomena, (190), 33-41.

http://dx.doi.org/10.1016/j.elspec.2013.02.001

Copyright: Elsevier

http://www.elsevier.com/

Postprint available at: Linköping University Electronic Press

http://urn.kb.se/resolve?urn=urn:nbn:se:liu:diva-103884 


\title{
Photoelectron spectroscopy and modeling of interface properties related to organic photovoltaic cells
}

\author{
M, Fahlman, ${ }^{1 *}$ P. Sehati, W. Osikowicz, ${ }^{1 * *}$ S. Braun, ${ }^{1}$ M.P. de Jong, ${ }^{2}$ and G. Brocks ${ }^{3}$ \\ ${ }^{1}$ Department of Physics, Chemistry and Biology, Linkoping University, SE-581 83 Linkoping, Sweden. \\ ${ }^{2}$ MESA+ Institute for Nanotechnology, University of Twente, P.O. Box 217, 7500 AE Enschede, The \\ Netherlands. \\ ${ }^{3}$ Computational Materials Science, Faculty of Science and Technology and MESA+ Institute for \\ Nanotechnology, University of Twente, P.O. Box 217, 7500 AE Enschede, The Netherlands. \\ * Corresponding author \\ *** Current address: Sapa Technology, S-61281 Finspang, Sweden
}

\begin{abstract}
In this short review, we will give examples on how photoelectron spectroscopy (PES) assisted by models on interface energetics can be used to study properties important to bulk heterojunction type organic photovoltaic devices focusing on the well-known bulk heterojunction blend of poly(3hexylthiophene):[6,6]-phenyl-C61-butyric acid methyl ester (P3HT:PCBM) and its model system P3HT: $\mathrm{C}_{60}$. We also will discuss some of the limitations of PES as applied to organic semiconductors (OS) and photovoltaic devices and finish with reviewing recent theoretical advances that now enable calculation of relevant parameters at (hybrid) interfaces measured by PES.
\end{abstract}

Keywords: Photoelectron spectroscopy, photovoltaics, organic semiconductors, interfaces

\section{Introduction}

It has been demonstrated that organic semiconductors can be used e.g. in light emitting diodes (OLEDs) [1], photovoltaic devices (OPV) [2] and in spintronic devices [3,4]. OS molecules and polymers offer some attractive advantages such as solution-based low-temperature processing ("electronic ink"), tunable light absorption/emission from near UV into IR (optimized coverage of the solar spectrum or visible spectrum), tunable ionization potentials and electron affinity (optimized charge injection or exciton dissociation at heterojunctions), and comparatively long spin life times (important for spintronics applications). OLEDs are now commercial and e.g. have seized the high-end market for cell phone displays. The field of organic photovoltaics has seen an increased rate of development in recent years with an improved understanding of the device physics [5-9] and improved device efficiency. The best confirmed terrestrial cell efficiencies in fact are now roughly on par with the more mature dye-sensitized solar cell (DSSC) technology [10]. Results from Mitsubishi, for example, using OS molecules and the well-established bulkheterojunction concept $[11,12]$ demonstrated a solar cell of $10 \%$ efficiency as compared to $11 \%$ (Sharp) and $9.9 \%$ (Sony) for dye-sensitized solar cells [10].

All organic-based electronic/spintronic devices are made by deposition of successive layers (metal, oxide, insulating or semiconducting layers), and many key electronic processes (such as charge/spin injection from metallic electrodes, charge recombination into light or light conversion into charges, etc.) occur at interfaces. In fact, as charges are localized on (parts of) molecules in most films, even charge/spin transport can be seen as a sequence of charge/spin injection events at organic-organic 
junctions. Organic electronic device performance thus is strongly linked to the energy level alignment at the various interfaces contained within, and it is of great importance to understand and predict energy level alignment at both metal-organic and organic-organic interfaces. Photoelectron spectroscopy (PES) has been used widely to this affect $[13,14]$, as it offers the ability to probe the density of occupied states (DOS) of a material, the ionization potential (IP) as well as the work function. In particular the change in work function, if any, at heterojunctions can be measured by PES, as can energy level gradients over a film, i.e., "band bending" type effects. It also has been shown that the equilibrium energetics at interfaces featuring OS molecules to a large extent can be explained and predicted by the Integer Charge Transfer (ICT) model [15-19] a model that is applicable to weakly-interacting interfaces such as metal-organic junctions prepared under ambient or high vacuum conditions where direct overlap of the organic $\pi$-system with the metal bands is prevented due to presence of oxides and/or hydrocarbons on the metal substrate, as well as for organic-organic heterojunctions, i.e., exactly the type of interfaces present in bulk heterojunction OPVs. Abrupt transitions between a Schottky-Mott regime and Fermi-level pinning regimes are predicted and observed upon variations of the work function of the substrate for both metal/organic and organic/organic interfaces. The Schottky-Mott regime is defined by vacuum level alignment at the interface while the Fermi-level pinning regimes are characterized with an interface potential step that scales with the difference between the equilibrium ionization potential or electron affinity of the organic semiconductor at the interface and the work function $\left(\mathrm{W}_{\mathrm{S}}\right)$ of the substrate. The origin of the potential step $(\Delta)$ is explained by spontaneous charge transfer across the interface via tunneling (integer charge transfer) [15-17] when the substrate work function is higher than the smallest energy required to take away one electron (lower than the largest energy gained from adding one electron) from (to) the molecule at an interface producing a fully relaxed state. These energies are referred to as $\mathrm{E}_{\mathrm{ICT}+}$ and $\mathrm{E}_{\mathrm{ICT}-}$, respectively. Three distinct energy level alignment regimes are hence predicted by the model: (i) $\mathrm{W}_{\mathrm{S}}>\mathrm{E}_{\mathrm{ICT}+}-$ Fermi level pinning to a positive integer charge transfer state, resulting in a substrate-independent work function; (ii) $\mathrm{E}_{\mathrm{ICT}-}<\mathrm{W}_{\mathrm{S}}<\mathrm{E}_{\mathrm{ICT}+}-$ Vacuum level alignment, giving a substrate-dependent work function, slope $=1$; (iii) $\mathrm{W}_{\mathrm{S}}<\mathrm{E}_{\mathrm{ICT}}$ - Fermi level pinning to a negative integer charge transfer state, resulting again in a substrate-independent work function, see Fig. 1. The importance of the physical nature of the interface in terms of inter- and intramolecular order was highlighted early on in the development of the ICT model, as it was expected to significantly affect the $\mathrm{E}_{\mathrm{ICT}+\text {.- }}$ values [16].

Bulk heterojunction type OPV devices are based on a network of donoracceptor type organic-organic heterojunctions created through blending of two (or more) organic components in a film sandwiched between two electrodes where at least one is transparent. The excitons created upon absorption of photons in such a film (by either the donor or acceptor molecules) should ideally be transferred to a donor/acceptor interface where they are transformed into electron-hole charge transfer complexes. These eventually become free negative and positive charge carriers, which are subsequently transported to the electrodes, see Fig. 2 [20]. Far from all excitons created follow this ideal path as a variety of loss mechanisms exist [20], as depicted in Fig. 2. The energy level off-set at the heterojunctions, e.g., should be large enough to overcome the exciton binding energy so as to assist the transformation of an exciton into a charge transfer complex, but if the off-set between the donor and acceptor levels is too large, substantial energy loss occurs and the overall power 
conversion efficiency of the cell will suffer [21]. The energetics at the OS/electrode interfaces also are important as barriers towards hole- and/or electron-extraction will decrease the open circuit voltage $\left(\mathrm{V}_{\mathrm{OC}}\right)$ and the overall power efficiency $(\eta)$ of the device, as the latter depends on $\mathrm{V}_{\mathrm{OC}}$, short circuit current $\left(\mathrm{J}_{\mathrm{SC}}\right)$, fill factor $(\mathrm{FF})$ and power of the incident irradiation $\left(\mathrm{P}_{0}\right)$ as $\eta=\mathrm{J}_{\mathrm{SC}} \mathrm{V}_{\mathrm{OC}} \mathrm{FF} / \mathrm{P}_{0}$. From this formula it is then clear that potential losses in a device should be minimized through optimization of the energetics at all interfaces.

A number of models for estimating the $\mathrm{V}_{\mathrm{OC}}$ of an OPV has been proposed [59], and it has been shown that for OPV devices with optimized electrode interfaces (no barriers for hole and electron extraction at donor/cathode and acceptor/anode interfaces, respectively), the $\mathrm{V}_{\mathrm{OC}}$ for a particular blend combination can be determined from the free-energy difference between the (first) excited charge transfer complex and the ground state charge transfer complex, which in turn can be measured by optical spectroscopy methods [8]. Though PES and inverse PES derived values for donor $\mathrm{h}^{+}$-polarons and acceptor $\mathrm{e}^{-}$-polarons can be used in models to predict $\mathrm{V}_{\mathrm{OC}}$, either directly [22] or by substituting cyclic voltammetry based estimates [9] of the same properties, the approach is certainly more expensive and time consuming than the optical spectroscopy (or cyclic voltammetry) approach. Where PES measurements clearly do bring an added value is in mapping out the energy levels $\left(\mathrm{E}_{\mathrm{ICT}+,-}\right)$ that determine the potential step, if any, at interfaces [23, 24]. As noted, the electrode interfaces have to be optimized so as to minimize losses to the $\mathrm{V}_{\mathrm{OC}}$. Furthermore, a potential step at the bulk heterojunction donor-acceptor interface, though decreasing the $\mathrm{V}_{\mathrm{OC}}$, may in fact improve power efficiency by increasing the percentage of charge transfer complexes that dissociate into free charges as we will discuss in some greater detail later in this review.

\section{Experimental methodology}

This review assumes that the reader is familiar with photoelectron spectroscopy and we will not delve into the basics of the method. We will, however, briefly discuss some (but not all) issues particular to the study of OS and their interfaces in OPVs, as the PES-based approach of determining the energy level alignment in such multi-layer organic electronic devices comes with some weaknesses. The unoccupied density of states is not directly accessible, for example, though the binding energy of the most easily reduced states at an interface can be measured indirectly, using the ICT model. Hence, estimates of the electron affinity (EA) of an organic film are typically obtained using a combination of PES and optical absorption data, cyclic voltammetry or inverse photoemission. Another, perhaps less obvious problem is derived from the nature of the charge carrying species in OS films. In $\pi$-conjugated molecules/polymers, charge injection (and optical excitation) modifies both the electronic and the geometric structure of the materials: when adding an electron to the antibonding lowest unoccupied molecular orbital (LUMO) or taking an electron from the highest occupied molecular orbital (HOMO), the bonds of the molecule are reorganized to minimize the energy with the result that the IP and EA differ significantly from the HOMO and LUMO energies, respectively. Hence, the HOMO and LUMO energies of the neutral system are not identical with the charge carrying species (polarons) that are relevant for determining e.g. energy level alignment and charge injection barriers. The binding energy measured by PES also is not equal to the binding energy of the electrons in the neutral ground state but represents the energy difference between the initial ground state and a final (excited) 
ionized state. However, the PES derived ionization potential typically is still not equal to the hole-polaron formation energy of the OS as the electron in a photoelectron emission event leaves a molecule typically within about $10^{-15} \mathrm{sec}$ whereas the bond relaxation time is around $10^{-13} \mathrm{sec}$ and the corresponding electronic relaxation time is about $10^{-16} \mathrm{sec}$ [25]. Thus, the electrons have had time to relax, i.e., the hole is fully screened, but the bonds (nuclei) are frozen during the photoemission process. This means that for systems where the ionized final state energy is significantly affected (lowered) by geometric relaxation (i.e. polarons), which is the case for most OS films, PES measurements will overestimate the hole-polaron formation energy and binding energy in general. Nevertheless, the PES-derived IP, representing the vertical IP and typically defined as the binding energy vs. vacuum level of the leading edge of the frontier feature in the valence region spectrum, is often (incorrectly) equated with the binding energy of the hole-transporting species (polarons) and the hole-injection barrier in e.g. diodes are then concomitantly estimated as the difference between the (effective) work function of the electrode and the IP of the organic film. This is problematic, not only because the PES IP does not take into account geometrical relaxation contributions to the polaron energy, but also because different types of devices and biasing conditions utilize different parts of the (polaron) DOS: e.g. a hybrid organic spin-valve is generally operated at orders of magnitude lower current densities than a light emitting diode, and field effect transistors (FET) operate at different fields and carrier concentrations than diode based devices. Hence, defining the energy position of the (polaron) DOS that is relevant to the OPV device from the PES spectrum alone is a daunting task.

PES measurement of the work function, and in extension the interface potential step (if any) and potential gradients over the films (if any), is more robust than the IP and EA ( $\mathrm{h}^{+}$- and $\mathrm{e}^{-}$-polarons, respectively) determination. The PES spectra of the valence region can provide valuable information not only about details of the electronic structure of the studied material but also about its work function $\mathrm{W}$ and the change in the work function $(\Delta)$ that can occur upon (partial) coverage of the substrate surface, as illustrated in Fig. 3. The work function can be obtained from the measured energy of the secondary electron cutoff $\left(\mathrm{E}_{\text {cutoff }}\right)$ (see Fig. 3) using a simple formula:

$$
\mathrm{W}=\mathrm{h} v-\mathrm{E}_{\text {cutoff }} \text {. }
$$

The change in work function, $\Delta$, can be monitored by remeasuring the $\mathrm{E}_{\text {cutoff }}$ after a deposition step has been carried out (spin-coating a polymer film, in situ condensation of a molecular (sub)monolayer, etc). Hence, the relation between the occupied levels at a heterojunction can be measured, both in reference to the vacuum level and the Fermi level, though within the limitations discussed above.

\section{Experimental results and discussion}

The field of OPV is currently pursuing the development of new materials for more efficient bulk-heterojunction solar cells, but the blend poly (3hexylthiophene):[6,6]-phenyl-C61-butyric acid methyl ester (P3HT:PCBM) still provides reasonable power efficiency and serves as an important model system [24, 26-30]. The P3HT:PCBM blend provides efficient charge generation and transport with low recombination and understanding of the underlying reasons for these advantageous properties aid the development of new material systems. As noted, it is of great importance to know the energetics at the various interfaces, in particular at the bulk donor-acceptor heterojunction. It is not easy to prepare a monolayer thick 
film from spin-coating, however, so we will first explore the $\mathrm{C}_{60} / \mathrm{P} 3 \mathrm{HT}$ interface to gain a deeper understanding of the bulk heterojunction energetics. $\mathrm{C}_{60}$ is a good model molecule for PCBM and can be deposited in well-controlled (sub)monolayer steps in situ, enabling the interface energetics to be studied in some detail.

\section{The $\mathrm{C}_{60} / \mathrm{P} 3 \mathrm{HT}$ heterojunction}

In the following section, we address the energy level alignment at the interface between regioregular poly(3-hexylthiophene), rr-P3HT, and fullerene $\mathrm{C}_{60}$ [31]. Rr-P3HT exhibits moderate electron donor character, due to its low vertical ionization potential (IP) of 4.5-4.6 eV as measured by PES. $\mathrm{C}_{60}$ is an electron acceptor, with an electron affinity (EA) of $4.0 \mathrm{eV}$ [32]. The $\mathrm{E}_{\mathrm{ICT}+}$ value for annealed rr-P3HT is $4.0 \mathrm{eV}$ [17] and the $\mathrm{E}_{\mathrm{ICT}-}$ value for $\mathrm{C}_{60}$ is $4.5 \mathrm{eV}$ [31], as derived from the ICT model applied to PES measurements, see Fig. 4. The $\mathrm{C}_{60} / \mathrm{P} 3 \mathrm{HT}$ interface was formed by (stepwise) deposition of $\mathrm{C}_{60}$ molecules in UHV, using a simple Knudsen-cell, onto a thin film $(20 \mathrm{~nm})$ of rr-P3HT. The latter was prepared by spin coating from a solution in dichlorobenzene, onto metallic or semiconducting substrates with selected work functions between 3.3 and $6.1 \mathrm{eV}$. This large range of work functions allows for a systematic study of the impact of the substrate work function on the energy level alignment throughout the full layer stack. This point is addressed in detail in ref. [31] but will not be discussed here.

Fig. 5 shows a typical example of the evolution of the valence band spectra recorded during stepwise $\mathrm{C}_{60}$ deposition (from 0 to $11 \mathrm{~nm} \mathrm{C}_{60}$ ) onto a rr-P3HT film (the same procedure was repeated for several rr-P3HT films on different substrates, yielding similar results). As indicated in the left panel of the figure the secondary electron cut-off shifts towards lower binding energies as $\mathrm{C}_{60}$ is deposited onto rrP3HT. This corresponds to a positive vacuum level shift $\Delta$, which in turn indicates the formation of a potential step at the interface, with its negative "pole" on the side of $\mathrm{C}_{60}$. The vacuum level shift saturates at a value of $0.6 \pm 0.1 \mathrm{eV}$ after deposition of about $5 \mathrm{~nm}$ of $\mathrm{C}_{60}$. It should be pointed out in passing that the effects of space charges induced by doping (impurities) cannot account for the observed vacuum level shift. Detailed studies of interfaces between $\mathrm{C}_{60}$ and atomically clean noble metal surfaces showed that noticeable space charge effects require film thicknesses as large as 100 $\mathrm{nm}$ for nominally $99.9 \%$ pure $\mathrm{C}_{60}$ [33] and can hence be ruled out here. The vacuum level shift must therefore be due to a different effect, namely the formation of an interface dipole due to transfer of electrons from P3HT to $\mathrm{C}_{60}$ at the interface. Charge transfer is indeed expected, given the values of the pinning levels of $\mathrm{P} 3 \mathrm{HT}$ and $\mathrm{C}_{60}$ and a $0.5 \mathrm{eV}$ shift in the vacuum level is predicted based on the $\mathrm{E}_{\mathrm{ICT}+,-}$ values, in good agreement with the experimental results.

The resulting charge transfer states, which appear in the energy gap of the organic semiconductors, generally are difficult to detect, especially for heterojunctions featuring a polymer overlayer, as (sub)monolayer deposition of polymers from spin coating is rarely achievable, as noted above. Furthermore, typically only a small fraction of charged species are involved in establishing equilibrium of the chemical potential across the interface, so even for a (sub)monolayer the signal from the charge transfer states may be hard to discern. Indeed, the raw UPS spectra shown in Fig. 5 do not reveal the presence of any charged $\mathrm{C}_{60}$ species (e $\mathrm{e}^{-}$-polarons). Nonetheless, by subtracting the signal of P3HT from the total UPS intensity in the spectra with nominal $\mathrm{C}_{60}$ overlayer thicknesses of $0.01 \mathrm{~nm}$ and $0.3 \mathrm{~nm}$ (note that the fraction of charged molecules is expected to be highest for low $\mathrm{C}_{60}$ coverage), clear evidence for the existence of negatively charged 
$\mathrm{C}_{60}$ molecules at the interface is found. This is demonstrated in Fig. 6, where a peak that corresponds to negatively charged $\mathrm{C}_{60}$ molecules, derived from the now singly occupied former LUMO, appears at $2.0 \mathrm{eV}$ above the HOMO of neutral $\mathrm{C}_{60}$. In addition, a broadening of $\mathrm{C}_{60}$ levels, as compared to the case of "bulk" $\mathrm{C}_{60}$, is observed in the very first stages of interface formation: The full width at half maximum (FWHM) of the $\mathrm{C}_{60} \mathrm{HOMO}$ level changes from approximately $0.7 \mathrm{eV}$ at the first deposition step to $0.5 \mathrm{eV}$ for a thick $\mathrm{C}_{60}$ overlayer. This may be attributed to lateral dipole field inhomogenities, which are indeed expected for a low charge density (a small fraction of charged/neutral molecules).

Hence, by using the $\mathrm{C}_{60} / \mathrm{P} 3 \mathrm{HT}$ model system, we have demonstrated that the interface energetics can be predicted from the $\mathrm{E}_{\mathrm{ICT}+,-}$ values determined by PES, which in turn enables choice of electrodes that minimize losses due to barriers. We further demonstrated that the formation of a ground state charge transfer complex can be predicted as well as verified by PES measurements. In the case of the $\mathrm{C}_{60} / \mathrm{P} 3 \mathrm{HT}$ interface, the formation of a ground state charge transfer complex induces an $0.6 \mathrm{eV}$ vacuum level shift at the heterojunction. We can thus expect a similar ground state charge transfer complex to form at the PCBM/P3HT heterojunction. This particular study focused on annealed rr-P3HT as the P3HT:PCBM blends are typically annealed in OPV devices in order to improve power efficiency. We will in the next section show how PES can be used to probe annealing-related effects on IP and $\mathrm{E}_{\mathrm{ICT}}$ values, and in extension, shed light on the positive effects a charge transfer complex induced potential step at the bulk heterojunction may provide.

\section{The PCBM/P3HT heterojunction}

To show how PES can be used to clarify the effect of annealing on the electronic structure and film morphology in OPV devices, and in turn how these properties may affect interface energetics and device efficiency, we describe PES measurements using HeI light performed on rr-P3HT thin films on Au [34], and again we interpret the results using the ICT. As previously described, the vertical ionization potential obtained by the PES technique includes the inter- and intra-molecular electronic relaxation that occurs in response to the creation of a hole in a molecular orbital and thus captures the electronic part of the polaronic relaxation energy in $\pi$ conjugated materials as well as the screening of the surrounding medium. Tracking changes in film morphology by following the evolution of the PES-derived IP is thus a viable method that has been employed previously on alkylsubstituted polythiophenes [35-37], where e.g. improved order in the polythiophene films will lead to better intermolecular screening and hence lower IP. Note that the PES-derived IP represents the vertical ionization potential of the material in the probed surface region and hence does not yield information on the local order at a buried interface, nor does it represent the true polaronic formation energy. The ICT model can be used in combination with PES data, however, to determine the energy of the integer charge transfer states $\mathrm{E}_{\mathrm{ICT}+,-,}$, [15-17] defined as the lowest energy required to take away one electron (highest energy gained from adding one electron) from (to) the molecule/polymer at an interface producing a fully relaxed polaron. Note that both electronic and geometrical relaxation are included in the $\mathrm{E}_{\mathrm{ICT}+}$ and $\mathrm{E}_{\mathrm{ICT}-}$, as well as effects from inter- and intra-molecular order at the interface [15-17, 23, 34], which may differ from the bulk and surface as shown below.

The annealing-induced evolution of the IP and $\mathrm{E}_{\mathrm{ICT}+}$ of rr-P3HT films is listed in Table 1 [34]. For the pristine rr-P3HT films, we see a low IP of $4.5 \mathrm{eV}$ and an $\mathrm{E}_{\mathrm{ICT}+}$ of $4.4 \mathrm{eV}$. The IP is significantly lower than that of regiorandom (rra-)P3HT, $4.65 \mathrm{eV}$ 
[34], indicating comparatively well delocalized polarons [34, 38, 39] due to longer intrachain $\pi$-conjugation lengths and better screening from neighboring chains as the interchain order is superior in the case of rr-P3HT. The PES data are thus in agreement with literature where pristine rr-P3HT films are defined by excellent interand intrachain order (2D lamellar structure) [38, 40-43]. Upon annealing, we see no increase in IP, in stark contrast to the behaviour of rra-P3HT where increased temperature induces significant interring torsion and increases the IP and band gap $[34,35]$. This suggests that the crystallinity of the rr-P3HT films prevents the chains inside of the grains from undergoing interring torsion as it would disrupt the tight $\pi-\pi$ packing of the polymer chains: the chains are collectively locked in place. The chains at the edges of the grains, however, are comparatively free to move, and some of these chains undergo thermal-induced increases in inter-ring torsion and hence decreases in the $\pi$-conjugation length, modification of the interchain order as well as their orientation in respect to the interface: all of which affects the $\mathrm{E}_{\mathrm{ICT}+}$ value. These chains dominate the interface energetics with the substrate and again the Fermi level gets pinned to their $\mathrm{E}_{\mathrm{ICT}+}$ states of around $4.05 \mathrm{eV}$. Such a decrease of $\mathrm{E}_{\mathrm{ICT}+}$ is likely due to a change in the orientation of (part of) the chains at the interface. The same value, $\mathrm{E}_{\mathrm{ICT}+}=4.05 \mathrm{eV}$, is achieved for thermally treated rra-P3HT, which is expected as the interface energetics is dominated by the chains with orientations that give the lowest pinning level. Cooling to room temperature does not return all of these chains to their pristine conformation, so the Fermi level remains pinned to the lower value, even though the bulk (and surface) polarons retain their delocalized nature, which is possibly even improved by an annealing-induced increase of the macroscopic order in the films as the IP is slightly lowered. Translating these results to P3HT:PCBM blends, we see that annealing in the rr-P3HT case creates a ground state charge transfer complex at the P3HT:PCBM interface with the negative side of the dipole pointing into the PCBM acceptor layer, as annealing at $110^{\circ} \mathrm{C}$ or above introduces chains at the interface with $\mathrm{E}_{\mathrm{ICT}+}$ values low enough to promote spontaneous charge transfer to the PCBM ( $\mathrm{E}_{\mathrm{ICT}}-=4.2 \mathrm{eV}$ [23], see Fig. 4), which is not the case for the as prepared RT case (rr-P3HT $\mathrm{E}_{\mathrm{ICT}+}=4.4 \mathrm{eV}>4.2 \mathrm{eV}$, hence vacuum level alignment).

Theoretical models predict that an interface dipole with the negative pole in the acceptor layer will enhance dissociation of charge transfer complexes into free charge carriers, significantly decreasing the chance that the electron and hole states become trapped at the interface by Coulomb forces where they eventually would recombine resulting in a loss of photocurrent [44, 45]. Furthermore, the charge transfer process described by the ICT model will sample the most easily oxidized polymer chains or chain segments on the P3HT side of the heterojunction, and the most easily reduced PCBM molecules at the other side. In this way, the most tightly bound charge transfer complexes that can be created at the interface are already occupied in the (dark) ground state and are consequently not available to participate in the exciton dissociation process following a photon absorption event [23].

Photoinduced absorption spectroscopy carried out by the Österbacka group of Åbo Akademi, see Table 2 [34], tested these predictions by following two different routes to charge transfer complex generation at rr-P3HT:PCBM bulk heterojunctions: above-bandgap excitation $(514 \mathrm{~nm})$ where the excitons migrates to a donor/acceptor interface and minimizes its energy by transforming in to a charge transfer complex or sub-bandgap excitation $(785 \mathrm{~nm})$ where the charge transfer complex is formed from direct excitation at the interface. For above-bandgap excitation, the concentration of free charges was found to increase significantly after heat treatment, and at the same time the lifetimes are significantly reduced. The results indicate that in rr-P3HT the 
well-ordered (lamellar) structure produces efficient delocalization and high mobility of the polarons. Charges can escape fairly well from the interfaces already before heat treatment, but the introduction of the interface dipole after heat treatment makes this escape process much more efficient resulting in higher charge concentration [34]. The relative increase in charge concentration is even bigger for sub-bandgap excitation, which may seem counterintuitive given that sub-bandgap excitation can only occur at the donor/acceptor interface and heat treatment of rr-P3HT:PCBM blends promote de-mixing and hence a significantly decreased interface area. However, as the most tightly bound charge transfer complexes available are already populated in the (dark) ground state after heat treatment according to the PES results and ICT-based interpretation thereof, generating the advantageous interface dipole, the direct excitation now produces charge transfer complexes with a significantly greater chance of dissociating, producing a net increase of free charges.

\section{Theoretical modeling}

As earlier alluded to, PES is an expensive technique and like all experimental techniques requires synthesis of the materials to be tested/screened for suitability in OPV devices, adding further cost and complexity. It would thus be desirable to replace some if not all PES-based measurements of the interface energetics phenomena described so far in this review with calculations, the latter being generally cheaper and do not require actual (time consuming) synthesis of the molecules/polymers to be tested. Recent advances in theory in fact show the promise of doing just that, as the $\mathrm{E}_{\mathrm{ICT}}$ values and in extension potential steps (if any) at (hybrid) organic interfaces now can calculated and reproduced to within the error margin of the PES experiments. Below we describe in some detail the density functional theory (DFT) based model [46-48], first for metal/OS then for OS heterojunctions and finally review some recent results.

Following the ICT model, we assume that the contact between the metallic electrode and the organic material is weak, implying that the electronic states of the organic molecules do not hybridize notably with those of the electrode. Electrochemical equilibrium between the electrode and the organic material is then attained by transferring (an integer number of) electrons across the metal-organic interface $[15,16]$. Electrostatic interactions keep the transferred charges, and their image charges in the metal, close to the interface. A "double layer" is formed at the interface, similar to those found at electrodes in electrochemical devices. The double layer leads to a potential step $\Delta$ across the metal-organic interface. In experiment such a potential step is often extracted as $\Delta=\mathrm{W}-\mathrm{W}_{\mathrm{S}}$, where $\mathrm{W}_{\mathrm{S}}$ is the work function of the electrode, in absence of the organic material, and $\mathrm{W}$ is the work function of the total system after deposition of the organic layer on the electrode.

Consider an interface between a metallic substrate and an organic layer of $\mathrm{N}$ acceptor molecules, and allow for transferring a number of electrons from the substrate to the acceptor layer. A particular configuration of the charges at the interface has the total energy

$$
\mathrm{E}\left(n_{1}, n_{2}, \ldots, n_{N}\right)=\sum_{i=1}^{N}\left[n_{i} E_{1, i}+\left(1-n_{i} E_{0, i}\right]+E_{C}\left(n_{1}, n_{2}, \ldots, n_{N}\right)\right.
$$

where $\mathrm{i}$ labels the molecules, $\mathrm{n}_{\mathrm{i}}=0,1$ is the occupation number of the molecular acceptor state, $E_{0, i}\left(E_{1, i}\right)$ is the total energy of a neutral (charged) molecule, and $E_{C}$ is the electrostatic energy of the interfacial arrangement of the charged molecules, all image charges in the substrate and polarization effects included. $\mathrm{E}_{\mathrm{C}}$ 
does not generally have a form that yields simple analytical expressions for configurational averages.

A simple model is obtained by assuming that the electrostatic energy of the interface can be described by a parallel plate capacitor

$$
E_{C}\left(n_{1}, n_{2}, \ldots, n_{N}\right) \approx E_{C}\left(N_{1}\right)=\frac{N_{1}^{2} e^{2}}{2 C}-N_{1} B^{-},
$$

with

$$
N_{1}=\sum_{i=1}^{N} n_{i}
$$

the total number of charged molecules at the interface, e the elementary charge, and $\mathrm{C}$ the capacitance of the interface. $\mathrm{B}^{-}$is the Coulomb energy associated with charging a single molecule with an electron. It is subtracted from the capacitor charging energy to avoid double counting, as per definition $\mathrm{B}^{-}$is already included in the total energy $E_{1, i}$ of a charged molecule.

To simplify the discussion, we have assumed that $\mathrm{B}^{-}$is independent of the molecule $\mathrm{i}$. If we also suppose that the electron affinity (A) is independent of the molecule i

$$
\mathrm{A}_{\mathrm{i}}=\mathrm{E}_{0, \mathrm{i}}-\mathrm{E}_{1, \mathrm{i}}=\mathrm{A}
$$

then the total energy is a function of the total number of charged molecules $\mathrm{N}_{1}$, rather than a function of the occupation numbers $\mathrm{n}_{\mathrm{i}}$ of the individual molecules

$\mathrm{E}\left(\mathrm{N}_{1}\right)=-\mathrm{N}_{1} \mathrm{~A}+\mathrm{E}_{\mathrm{C}}\left(\mathrm{N}_{1}\right)+\mathrm{E}_{0}$

with $\mathrm{E}_{0}=\sum_{i=1}^{N} \mathrm{E}_{0, \mathrm{i}}$ the ground state energy of the molecular layer.

We assume that the organic layer and the metallic substrate are in equilibrium, with the substrate acting as an electron reservoir at a chemical potential $\mu$. The average number of charged molecules at temperature $\mathrm{T}$ is then given by the standard expression

$$
\bar{N}_{1}=\frac{\sum_{N_{1}=1}^{N} N_{1} g\left(N_{1}\right) e^{-\beta\left[E\left(N_{1}\right)-\mu N_{1}\right]}}{\sum_{N_{1}=1}^{N} g\left(N_{1}\right) e^{-\beta\left[E\left(N_{1}\right)-\mu N_{1}\right]}},
$$

with $\beta=1 / \mathrm{k}_{\mathrm{B}} \mathrm{T}$, and $\mathrm{g}\left(\mathrm{N}_{1}\right)$ the degeneracy of the energy level $\mathrm{E}\left(\mathrm{N}_{1}\right)$. The latter is a quadratic expression in $\mathrm{N}_{1}$, so the Gibbs factor $\exp \left\{\beta\left[E\left(N_{1}\right)-\mu N_{1}\right]\right\}$ is a Gaussian function. If we assume that the energy levels are non-degenerate, i.e. $\mathrm{g}\left(\mathrm{N}_{1}\right)$ $=1$, then the average $\bar{N}_{1}$ is derived from $\mathrm{E}\left(\bar{N}_{1}\right)-\mu \bar{N}_{1}=0$, giving

$$
\Delta \equiv \frac{\bar{N}_{1} e^{2}}{C}=W_{I C T}^{-}+\mu,
$$

with

$$
W_{I C T}^{-}=A+B^{-},
$$


The schematic energy level diagram is given in Fig. 1, where the $\mathrm{W}_{\text {ICT }}$ are equivalent to the corresponding experimentally derived $\mathrm{E}_{\mathrm{ICT}}$. Clearly $\Delta$ is the potential step across the interface, derived from the charges in the molecular layer and the screening charges in the substrate, as modeled by a parallel plate capacitor. As the work function of the clean substrate without the molecular layer is $\mathrm{W}_{\mathrm{S}}=-\mu$, the work function of the total system, including the molecular layer, is given by

$$
W=W_{S}+\Delta=W_{I C T}^{-},
$$

The work function is fixed by the molecular layer and is independent of the work function of the substrate, which is called work function pinning, or Fermi level pinning. Note that by construction $\bar{N}_{1} \geq 0$ and thus $\Delta \geq 0$. It also follows that, if $\mathrm{W}_{\mathrm{S}}>$ $W_{I C T}^{-}$, then $\bar{N}_{1}=0$ and thus $\Delta=0$. In that case $\mathrm{W}=\mathrm{W}_{\mathrm{S}}$, which is the Schottky-Mott limit. Upon increasing the substrate work function $\mathrm{W}_{\mathrm{S}}$, one observes a transition from pinning to Schottky-Mott behavior, the transition point being at $\mathrm{W}_{\mathrm{S}}=W_{I C T}^{-}$.

The assumption of non-degenerate energy levels leads to a temperature independent work function W. Non-degeneracy occurs if strong correlations between the charges in the molecular layer force these charges to assume a single particular geometric arrangement, which may not be an entirely realistic assumption. An opposing starting-point is to assume that the charges within the layer are completely uncorrelated, i.e. that every configuration of $\mathrm{N}_{1}$ charges within the layer of $\mathrm{N}$ molecules yields the same energy. The degeneracy of the energy levels is then given by

$$
g\left(N_{1}\right)=\frac{N !}{N_{1} !\left(N-N_{1}\right) !} 2^{N_{1}}
$$

where the factor $2^{N_{1}}$ accounts for the spin degeneracy. An expression for $\bar{N}_{1}$, and thus for $\Delta$ follows from the standard procedure of statistical physics, assuming that both $\mathrm{N}$ and $\mathrm{N}_{1}$ are large [49],

$$
\Delta=W_{I C T}^{-}+\mu+k_{B} T \ln \left\lceil\frac{2\left(\Delta_{M}-\Delta\right)}{\Delta}\right\rceil,
$$

where

$$
\Delta_{M}=\frac{N e^{2}}{C}
$$

is the maximal potential step at the interface, when all molecules are charged. The last term on the right-hand side represents the effect of temperature. We estimate $\Delta_{\mathrm{M}}$ to be typically in the range $10-20 \mathrm{eV}$ for organic layers adsorbed on metallic substrates. As a typical value of an interface potential step $\Delta$ is $1 \mathrm{eV}$, this means that at room temperature $\Delta$ is increased by $\sim 0.1 \mathrm{eV}$, as compared to its zero temperature value. Such a change is close to the error bar in photoemission experiments, and will be difficult to measure. Moreover, the assumption of completely uncorrelated charges may also not be entirely realistic, so the effect of temperature may be smaller than this.

Eq. (12) can be rewritten in a form that expresses the Fermi-Dirac statistics of the electrons more explicitly [50] 


$$
\bar{n}=\frac{\Delta}{\Delta_{M}}=\frac{1}{\frac{1}{2} e^{\beta\left(\Delta-W_{I C T}^{-}-\mu\right)}+1}
$$

with $\bar{n}$ the average occupation number of each molecule. This expression also demonstrates the weak temperature dependence more explicitly; if $\Delta-W_{I C T}^{-}-\mu \gg$ $\mathrm{k}_{\mathrm{B}} \mathrm{T}$, then the function at right-hand side is close to the step function $1-\Theta\left(\Delta-W_{I C T}^{-}-\right.$ $\mu$ ), and the solution to this equation is given by Eq. (10).

As the effect of temperature is not very large, we can to a good approximation replace $\Delta$ on the right-hand side of Eq. (12) by $W_{I C T}^{-}+\mu$. For the work function of the total system we then obtain

$$
W \approx W_{I C T}^{-}+k_{B} T \ln \left[\frac{2\left(\Delta_{M}-W_{I C T}^{-}+W_{S}\right)}{W_{I C T}^{-}-W_{S}}\right]
$$

using $\mu=-\mathrm{W}_{\mathrm{S}}$ as before. The work function $\mathrm{W}$ is not completely pinned, i.e. independent of $\mathrm{W}_{\mathrm{S}}$. However, using a typical value $\Delta_{\mathrm{M}}=15 \mathrm{eV}$ and decreasing $W_{I C T}^{-}$$\mathrm{W}_{\mathrm{S}}$ from 2 to $1 \mathrm{eV}$, increases $\mathrm{W}$ by $\sim 0.05 \mathrm{eV}$ at room temperature. This indicates that within the error bar of photoemission experiments, the work function can be considered as pinned. The main effect of temperature is to round off the transition from work function pinning to Schottky-Mott behavior for small values of $W_{I C T}^{-}-\mathrm{W}_{\mathrm{S}}$, upon increasing $\mathrm{W}_{\mathrm{S}}$.

So far we discussed electron transfer to acceptor molecules. A model for electron transfer from a layer of donor molecules to a metallic substrate can be developed in an analogous way. The total energy of Eq. (2) is then

$$
E\left(N_{1}\right)=N_{1} I+\frac{N_{1}^{2} e^{2}}{2 C}-N_{1} B^{+}+E_{0},
$$

with I the ionization potential of the molecules, and $\mathrm{B}^{+}$the Coulomb energy associated with charging a single molecule with a hole. Assuming completely uncorrelated electrons, leading to a degeneracy as in Eq. (11), and following the procedure outlined above, then yields for the work function of the total system

$$
W \approx W_{I C T}^{+}-k_{B} T \ln \left[\frac{2\left(\Delta_{M}+W_{I C T}^{+}-W_{S}\right)}{W_{S}-W_{I C T}^{+}}\right]
$$

with

$$
W_{I C T}^{+}=I-B^{+}
$$

the pinning level for holes. The resulting schematic energy level diagram is the same as in Fig. 1, with the theoretical $\mathrm{W}_{\text {ICT }}$ corresponding to the respective measured $\mathrm{E}_{\mathrm{ICT}}$. The potential step at the interface $\Delta=\mathrm{W}-\mathrm{W}_{\mathrm{S}}$ going from the metal to the organic layer, is negative, as the charges on the molecules are positive. So by construction $\Delta \leq 0$, and pinning to $W_{I C T}^{+}$occurs for substrates with a work function $\mathrm{W}_{\mathrm{S}}$ $>W_{I C T}^{+}$. As discussed above, we expect the temperature dependence of the work function $\mathrm{W}$ to be small, e.g. using $\mathrm{V}_{\mathrm{M}}=15 \mathrm{eV}$, room temperature, and increasing $\mathrm{W}_{\mathrm{S}}$ - $W_{I C T}^{-}$from 1 to $2 \mathrm{eV}$ increases $\mathrm{W}$ by $\sim 0.05 \mathrm{eV}$. The main effect of temperature is to round off the transition from Schottky-Mott behavior to work function pinning for small values of $\mathrm{W}_{\mathrm{S}}-W_{I C T}^{-}$upon increasing $\mathrm{W}_{\mathrm{S}}$. 
Following the experimentally-derived ICT-model, the work function pinning levels $W_{I C T, A}^{-}$, obtained for an individual layer of acceptor molecules, and $W_{I C T, D}^{+}$, obtained for an individual layer of donor molecules, can be used to predict the potential step $\Delta_{\mathrm{DA}}$ found at the interface between the donor layer and the acceptor layer

$$
\Delta_{D A} \approx W_{I C T, A}^{-}-W_{I C T, D}^{+}
$$

if $W_{I C T, A}^{-}>W_{I C T, D}^{+}$, and $\Delta_{\mathrm{DA}} \approx 0$, if $W_{I C T, A}^{-}<W_{I C T, D}^{+}$. It seems reasonable to assume that this potential step is caused by the transfer of electrons across the interface from the donor molecules to the acceptor molecules, for which we can use essentially the same approach as discussed above. The total energy is described by

$$
E\left(N_{1}\right)=N_{1} I_{D}-N_{1} A_{A}+\frac{N_{1}^{2} e^{2}}{2 C}-N_{1} B_{D}^{+}-N_{1} B_{A}^{-}+E_{0}^{\prime},
$$

where $I_{D}\left(A_{A}\right)$ is the ionization potential (electron affinity) of the donor (acceptor) molecule, $B_{D}^{+}\left(B_{A}^{-}\right)$is the Coulomb energy associated with charging a single donor (acceptor) molecule with a hole (electron), and $E_{0}^{\prime}$ the ground state energy of the neutral layers of donor and acceptor molecules. If we assume that the charges are strongly correlated, leading to non-degenerate energy levels, then the potential step is given by Eq. (19) and is temperature independent. For uncorrelated charges the degeneracy of the energy levels is given by

$$
g\left(N_{1}\right)=\left[\frac{N !}{N_{1} !\left(N-N_{1}\right) !}\right]^{g_{e}} g_{e}^{2 N_{1}}
$$

Here $\mathrm{g}_{\mathrm{e}}=1$ if we assume that an electron transferred from a donor to an acceptor molecule stays close to the hole left on the donor molecule, and the two form a singlet state. Alternatively, $\mathrm{g}_{\mathrm{e}}=2$ if we assume that the holes and the electrons in the donor and acceptor layers are completely uncorrelated, and both have spin degeneracy. Following the procedure outlined above, we then find

$$
\Delta_{D A} \approx W_{I C T, A}^{-}-W_{I C T, D}^{+}+g_{e} k_{B} T \ln \left[\frac{g_{e}^{2}\left(\Delta_{M}-W_{I C T, A}^{-}+W_{I C T, D}^{+}\right)}{W_{I C T, A}^{-}-W_{I C T, D}^{+}}\right],
$$

if $W_{I C T, A}^{-}>W_{I C T, D}^{+}$, and $\Delta_{\mathrm{DA}} \approx 0$, if $W_{I C T, A}^{-}<W_{I C T, D}^{+}$. Measuring the temperature dependence of $\Delta_{\mathrm{DA}}$ might be a way to characterize the degree of correlation of the charges at the interface.

To calculate the pinning levels for electrons and holes in molecular layers, Eqs. (10) and (18), we need the electron affinity A, the ionization potential I, and the molecular charging energies $\mathrm{B}^{-/ 4}$. All these quantities can in principle be obtained from first-principles calculations, but some care must be taken, as they all depend strongly on the environment of the molecule. The charge on a molecule is screened by polarization of the molecules surrounding the charged molecule or image charges in the substrate. Screening stabilizes the charged molecule by an energy $\mathrm{P}^{-/ /}$for a negatively/positively charged molecule, which increases the electron affinity to $A^{\prime}=$ $\mathrm{A}+\mathrm{P}^{-}$, and decreases the ionization potential to $I^{\prime}=\mathrm{I}-\mathrm{P}^{+}$. The molecular charging 
energies decrease to $B^{\prime-/+}=\mathrm{B}^{-/ 4}-\mathrm{P}^{-/ 4}$ for the same reason, i.e. charged molecules are stabilized by screening.

This reasoning leads to the remarkable conclusion that the pinning levels $W_{I C T}^{-/+}$do not depend on screening by the environment. They are independent of the dielectric properties of the environment, including those of the substrate. That does not mean that the pinning levels are pure molecular properties, however, as arguments similar to those applied for screening (the effects resulting from an induced charge distribution) cannot be made for the effects that result from the static charge distribution of the environment. The fields originating from the multipoles of the molecules surrounding a particular donor or acceptor molecule affect the energy levels of that molecule. It is, for instance, well-known that these energy levels depend on the orientation of the molecule within a molecular layer [51, 52]. Varying the packing and orientation of molecules within a layer can easily change the molecular ionization potential by $\gtrsim 0.5 \mathrm{eV}$. These static effects affect the energy levels of both the neutral and the charged molecule, and they will in general not cancel out.

In principle the same argument can be made for the influence of the static fields originating from the substrate. One would however expect that a metallic substrate does not give rise to strong local fields, as these will be screened by the metal. In conclusion, the pinning levels are expected to be a property of the molecular layers, and can be obtained from first-principles calculations on individual layers of donor or acceptor molecules.

If we use density functional theory (DFT) with common local, or semi-local functionals, i.e. the local density approximation (LDA), or the generalized gradient approximation (GGA), further simplifying approximations can be made. Such functionals allow for partial occupation numbers $n_{k}$ of the Kohn-Sham energy levels $\xi_{\mathrm{k}}$, and yield an expression for the total energy of a molecule $\mathrm{E}_{\mathrm{mol}}$ that is analytical in these occupation numbers. Janak's theorem holds for such functionals, which expresses the energy levels as derivatives of the total energy with respect to the occupation numbers, $\xi_{k}=\partial E_{m o l} / \partial n_{k}[53,54]$. The vertical electron affinity $\left(\mathrm{A}_{v}\right)$ can then be found by occupying the Kohn-Sham LUMO level at fixed molecular geometry

$$
A_{v}=-\int_{0}^{1} \xi_{L U M O}(n) d n
$$

Assuming that $\xi_{\mathrm{LUMO}}(\mathrm{n})$ is described well by a linear function $\varepsilon_{\mathrm{LUMO}}+\mathrm{U}^{-n}$, with $\varepsilon_{\mathrm{LUMO}}=\xi_{\mathrm{k}}(0)$ the empty Kohn-Sham LUMO level of a neutral molecule, one obtains

$$
A_{v}=-\varepsilon_{L U M O}-\frac{1}{2} U^{-}
$$

The physical meaning of the parameter $\mathrm{U}^{-}$can be clarified by integrating Janak's expression for a molecule with a partially occupied LUMO within the same approximation

$$
E_{m o l}(n)=E_{m o l}(0)+\varepsilon_{L U M O} n+\frac{1}{2} U^{-} n^{2}
$$

The quadratic term on the right-hand side can be associated with a molecular charging energy for an electron. Applying this approximation it means 


$$
B^{-}=\frac{1}{2} U^{-}
$$

By the same reasoning it follows that the vertical ionization potential is given by

$$
I_{v}=-\varepsilon_{\text {Hомо }}+\frac{1}{2} U^{+}
$$

with $\varepsilon_{\mathrm{HOMO}}$ the filled Kohn-Sham HOMO level of a neutral molecule, and

$$
B^{+}=\frac{1}{2} U^{+}
$$

the molecular charging energy for a hole.

In calculating the pinning levels one needs the adiabatic electron affinity and ionization potential, as we assume that the system is in equilibrium, where the charged molecules are relaxed. Indicating the relaxation energies of the charged molecules by $E_{\text {rel }}^{-,+}$, the pinning level for electrons becomes

$$
W_{I C T}^{-}=-\varepsilon_{L U M O}+E_{r e l}^{-},
$$

and the pinning level for holes is

$$
W_{I C T}^{+}=-\varepsilon_{\text {HOMO }}-E_{\text {rel }}^{+}
$$

Note that both $\varepsilon_{\text {HOMO }}$ and $\varepsilon_{\text {LUMO }}$ can be obtained from a DFT calculation on a layer of neutral molecules. Only the calculation of $E_{\text {rel }}^{-,+}$involves charged molecules. These relaxation energies are however not very sensitive to the environment of a molecule. Moreover, for most molecules the relaxation energies are small, $E_{\text {rel }}^{-,+} \lesssim 0.1$ $\mathrm{eV}$, unless the HOMO or LUMO state is very localized (as in a small molecule, for instance), or the molecule is exceptionally floppy.

We finish this section by presenting some results where the pinning levels according to Eqs. (29) and (30) were calculated using the Vienna Ab Initio Simulation Package (VASP) [55, 56] with projector augmented waves (PAW) $[56,57]$ and the PW91 GGA functional [58, 59]. We used close-packed molecular monolayers with periodic boundary conditions and chose the unit cell in the direction perpendicular to the layers sufficiently large, so that the potential in the middle of the cell represents the vacuum level. The Kohn-Sham energy levels are calculated with respect to this energy level. Many planar conjugated molecules have crystal structures that are naturally comprised of molecular layers. The preferential growth direction in such crystals is along a layer, with the molecules $\pi$-stacked along the layer and the molecular planes making a small angle with the normal to the layer. It is reasonable to assume that molecules deposited on a substrate adapt a similar growth pattern as in a crystal, if the interaction of molecules with a substrate is smaller than the interaction between the molecules. We refer to the molecules in such layers as "standing" (s). Alternatively, if the interaction with the substrate dominates, the adsorbed molecules are expected to form a close-packed structure with the molecular planes parallel to the molecular layer and to the substrate. We refer to such molecules as "lying" (1). The 
results on some typical donor and acceptor molecules relevant to OPV devices are given in Table III $[46,48]$, showing excellent agreement with experimental results.

\section{Summary}

We have discussed the advantages and limitations of using photoelectron spectroscopy to measure properties relevant to bulk heterojunction organic photovoltaic devices. Though photoelectron spectroscopy can at best only provide the vertical ionization potential of the donor material and indirectly using either modeling or other experimental techniques the acceptor electron affinity, the method is excellent for determining vacuum level offsets (potential steps) at interfaces and can when assisted by models also predict such offsets through the use of so-called integer charge transfer states (pinning energies), thus aiding in the design of loss-less electrode contacts and optimized bulk heterojunctions. We further showed that the existence of a ground charge transfer complex at the bulk heterojunction can in fact improve device properties by enhancing the transformation of excitons into free charge carriers. Finally, we described a recently developed theoretical technique for calculating the pinning energies that enables the enables mapping out of the potential land scape across a multilayer stack in silico.

\section{Acknowledgements}

This review is partly based on work funded by the European project MINOTOR, grant no. FP7-NMP-228424, and by STEM, the Swedish Energy Agency.

\section{References}

[1] C.W. Tang and S.A. Vanslyke, Appl. Phys. Lett., 51 (1987) 913.

[2] C.W. Tang, Appl. Phys. Lett., 48 (1986) 183.

[3] V. Dediu, M. Murgia, F.C. Matacotta, C. Taliani, and S. Barbanera, Solid State Communications, 122 (2002) 181.

[4] Z.H. Xiong, D. Wu, Z. Valy Vardeny, and J. Shi, Nature, 427 (2004) 821-824.

[5] M.D. Perez, C. Borek, S.R. Forrest, and M.E. Thompson, J. Am. Chem. Soc., 131 (2009) 9281.

[6] W.J. Potscavage, A. Sharma, and B. Kippelen, Accounts of Chemical Research, 42 (2009) 1758.

[7] K. Vandewal, K. Tvingstedt, A. Gadisa, O. Inganas, and J.V. Manca, Nature Materials, 8 (2009) 904.

[8] K. Vandewal, K. Tvingstedt, A. Gadisa, O. Inganas, and J.V. Manca, Phys. Rev. B, $81(2010) 8$.

[9] D. Veldman, S.C.J. Meskers, and R.A.J. Janssen, Advanced Functional Materials, 19 (2009) 1939.

[10] M.A. Green, K. Emery, Y. Hishikawa, W. Warta, and E.D. Dunlop, Prog. Photovolt. Res. Appl., 20 (2012) 12.

[11] M. Hiramoto, H. Fujiwara, and M. Yokoyama, Appl. Phys. Lett., 58 (1991) 1062.

[12] M. Hiramoto, H. Fujiwara, and M. Yokoyama, J. ASppl. Phys., 72 (1992) 3781.

[13] H. Ishii, K. Sugiyama, E. Ito, and K. Seki, Adv. Mater., 11 (1999) 605.

[14] N. Koch, ChemPhysChem, 8 (2007) 1438.

[15] S. Braun, W.R. Salaneck, and M. Fahlman, Adv. Mater., 21 (2009) 1450.

[16] M. Fahlman, A. Crispin, X. Crispin, S.K.M. Henze, M.P.d. Jong, W. Osikowicz, C. Tengstedt, and W.R. Salaneck, J. Phys.: Condens. Matter, 19 (2007) 183202. 
[17] C. Tengstedt, W. Osikowicz, W.R. Salaneck, I.D. Parker, C.-H. Hsu, and M. Fahlman, Appl. Phys. Lett., 88 (2006) 053502.

[18] S. Braun, M.P.d. Jong, W. Osikowicz, and W.R. Salaneck, Appl. Phys. Lett., 91 (2007) 202108.

[19] S. Braun, X. Liu, W.R. Salaneck, and M. Fahlman, Org. Electron., 11 (2010) 212.

[20] B. Kippelen and J.L. Brédas, Energy Environ. Sci., 2 (2009) 251.

[21] L.J.A. Koster, V. D.Mihailetchi, and P.W.M. Blom, Appl. Phys. Lett., 88 (2006) 093511.

[22] B.P. Rand, D.P. Burk, and S.R. Forrest, Phys. Rev. B, 75 (2007) 115327.

[23] P. Sehati, S. Braun, L. Lindell, X. Liu, L.M. Andersson, and M. Fahlman, IEEE J. Sel. Top. Quantum Electron., 16 (2010) 1718.

[24] Z. Xu, L.M. Chen, M.H. Chen, G. Li, and Y. Yang, Appl. Phys. Lett., 95 (2009) 013301.

[25] C.S. Fadley, Basic Concepts of X-ray Photoelectron Spectrsocopy, in Electron Spectroscopy: Theory, Techniques and Applications, C.R. Bundle and A.D. Baker, Editors. 1978, Academic Press: London. p. 2.

[26] Z.L. Guan, J.B. Kim, H. Wang, C. Jaye, D.A. Fischer, Y.L. Loo, and A. Kahn, Org. Electron., 11 (2010) 1779.

[27] R.J. Davis, M.T. Lloyd, S.R. Ferreira, M.J. Bruzek, S.E. Watkins, L. Lindell, P. Sehati, M. Fahlman, J.E. Anthony, and J.W.P. Hsu, J. Mater. Chem., 21 (2011) 1721.

[28] N. Schmerl and G. Andersson, Phys. Chem. Chem. Phys., 13 (2011) 14993.

[29] E. Varene and P. Tegeder, Appl. Phys. A-Mater. Sci. Process., 107 (2012) 13.

[30] W.C. Tsoi, S.J. Spencer, L. Yang, A.M. Ballantyne, P.G. Nicholson, A. Turnbull, A.G. Shard, C.E. Murphy, D.D.C. Bradley, J. Nelson, and J.S. Kim, Macromolecules, 44 (2011) 2944.

[31] W. Osikowicz, M.P.d. Jong, and W.R. Salaneck, Adv. Mater., 19 (2007) 4213.

[32] R.W. Lof, M.A.v. Veenendal, B. Koopmans, H.T. Jonkman, and G.A. Sawatzky, Phys. Rev. Lett., 68 (1992) 3924.

[33] N. Hayashi, H. Ishii, Y. Ouchi, and K. Seki, J. Appl. Phys., 92 (2002) 3784.

[34] H. Aarnio, P. Sehati, S. Braun, M. Nyman, M.P.d. Jong, M. Fahlman, and Ronald Österbacka, 1 (2011) 792., Adv. Energy Mater., 1 (2011) 792.

[35] W.R. Salaneck, O. Inganäs, B. Thémans, J.O. Nilsson, B. Sjögren, J.-E. Österholm, J.L. Brédas, and S. Svensson, J. Chem. Phys. , 89 (1988) 4613.

[36] K. Kanai, T. Miyazaki, H. Suzuki, M. Inaba, Y. Ouchi, and K. Seki, Phys. Chem. Chem. Phys., 12 (2010) 273.

[37] K. Kanai, T. Miyazaki, T. Wakita, K. Akaike, T. Yokoya, Y. Ouchi, and K. Seki, Adv. Funct. Mater., 20 (2010) 2046.

[38] R. Österbacka, C.P. An, X.M. Jiang, and Z.V. Vardeny, 287 (2000) 839.

[39] D. Beljonne, J. Cornil, H. Sirringhaus, P.J. Brown, M. Shkunov, R.H. Friend, and J.-L. Brédas, Adv. Funct. Mater., 11 (2001) 229.

[40] H. Sirringhaus, P.J. Brown, R.H. Friend, M.M. Nielsen, K. Bechgaard, B.M.W. Langeveld-Voss, A.J.H. Spiering, R.A.J. Janssen, E.W. Meijer, P. Herwig, and D.M.d. Leeuw, Nature, 40 (1999) 685.

[41] R.M. Beal, A. Stavrinadis, J.H. Warner, J.M. Smith, H.E. Assender, and A.A.R. Watt, Macromolecules, 43 (2010) 2343.

[42] R.D. McCullough, S. Tristram-Nagle, S.P. Williams, R.D. Lowe, and M. Jayaraman, J. Am. Chem. Soc., 115 (1993) 4910.

[43] T.-A. Chen, X. Wu, and R.D. Rieke, J. Am. Chem. Soc., 117 (1995) 233. 
[44] S. Verlaak, D. Beljonne, D. Cheyns, C. Rolin, M. Linares, F. Castet, J. Cornil, and P. Heremans, Adv. Funct. Mater. , 19 (2009) 3809.

[45] V.I. Arkhipov, P. Heremans, and H. Bässler, Appl. Phys. Lett., 82 (2003) 4605.

[46] M. Bokdam, D. Cakir, and G. Brocks, Appl. Phys. Lett., 98 (2011) 113303.

[47] G. Brocks, D. Cakir, M. Bokdam, M.P. de Jong, and M. Fahlman, Org. Electron., 13 (2012) 1793.

[48] D. Cakir, M. Bokdam, M.P. de Jong, M. Fahlman, and G. Brocks, Appl. Phys. Lett., 100 (2012) 4.

[49] H. Gould and J. Tobochik, Statistical and Thermal Physics: With Computer Applications. 2010, Princeton: Princeton University Press.

[50] N.W. Ashcroft and N.D. Mermin, Solid State Physics. 1976, Philadelphia: HoltSaunders International Editions.

[51] S. Duhm, G. Heimel, I. Salzmann, H. Glowatzki, R.L. Johnson, A. Vollmer, J.P. Rabe, and N. Koch, Nature Materials, 7 (2008) 326.

[52] W.R. Salaneck, Phys. Rev. Lett., 40 (1978) 60.

[53] J.C. Slater, Adv. Quantum Chem., 6 (1972) 2.

[54] J.F. Janak, Phys. Rev. B, 18 (1978) 7165.

[55] G. Kresse and J. Hafner, Phys. Rev. B (R), 47 (1993) 558.

[56] G. Kresse and D. Joubert, Phys. Rev. B, 59 (1999) 1758.

[57] P.E. Blöchl, Phys. Rev. B, 50 (1994) 17953.

[58] J.P. Perdew, J.A. Chevary, S.H. Vosko, K.A. Jackson, M.R. Pederson, D.J. Singh, and C. Fiolhais, Phys. Rev. B, 46 (1992) 6671.

[59] J.P. Perdew, J.A. Chevary, S.H. Vosko, K.A. Jackson, M.R. Pederson, D.J. Singh, and C. Fiolhais, Phys. Rev. B, 48 (1993) 4978.

[60] I.G. Hill, D. Milliron, J. Schwartz, and A. Kahn, Appl. Surf. Sci., 166 (2000) 354.

[61] H.Y. Mao, F. Bussolotti, D.-C. Qi, R. Wang, S. Kera, N. Ueno, A.T.S. Wee, and W. Chen, Org. Electron., 12 (2011) 534.

[62] H. Peisert, A. Petr, L. Dunsch, T. Chassé, and M. Knupfer, ChemPhysChem, 8 (2007) 386.

[63] R.J. Murdey and W.R. Salaneck, Jpn J Appl Phys Part 1 Regul Pap Short Note Rev Pap, 44 (2005) 3751. 


\section{Figures}

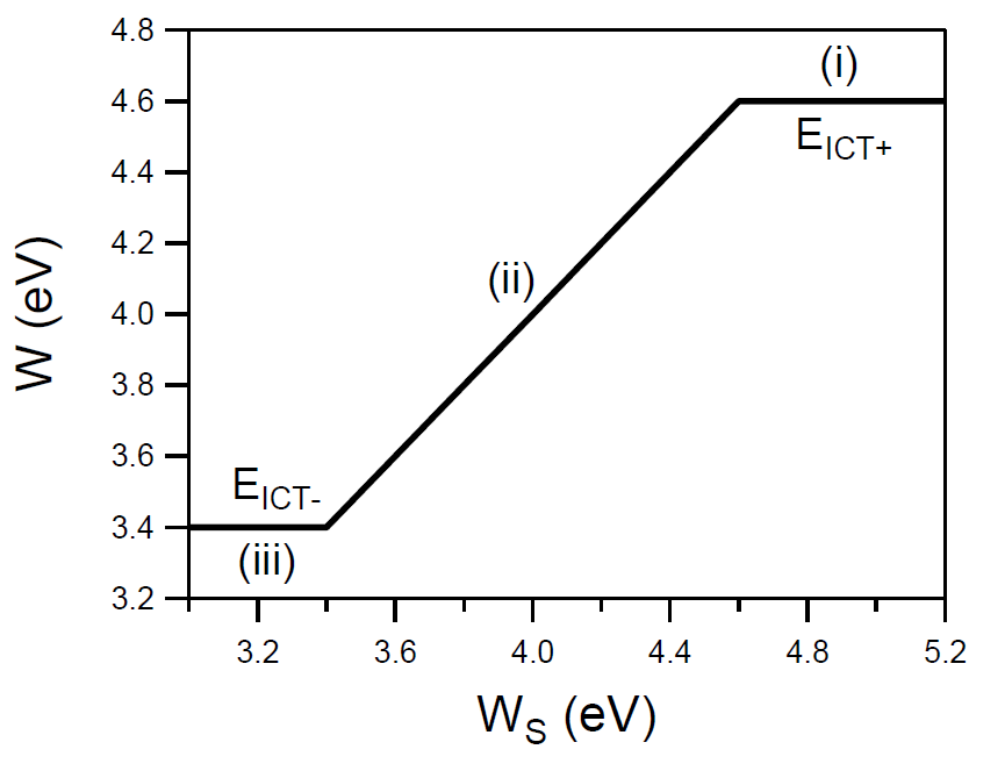

Figure 1. General diagram of the substrate work function $\left(\mathrm{W}_{\mathrm{S}}\right)$ dependence of the final work function (W) for $\pi$-conjugated organic molecules and polymers films deposited onto weakly-interacting surfaces. Three distinct regimes are present: (i) $\mathrm{W}_{\mathrm{S}}$ $>\mathrm{E}_{\mathrm{ICT}+}$ : Fermi level pinning to a positive integer charge transfer state, (ii) $\mathrm{E}_{\mathrm{ICT}-}<\mathrm{W}_{\mathrm{S}}$ $<\mathrm{E}_{\mathrm{ICT}+}$ : Vacuum level alignment, and (iii) $\mathrm{W}_{\mathrm{S}}<\mathrm{E}_{\mathrm{ICT}-}$ : Fermi level pinning to a negative integer charge transfer state.

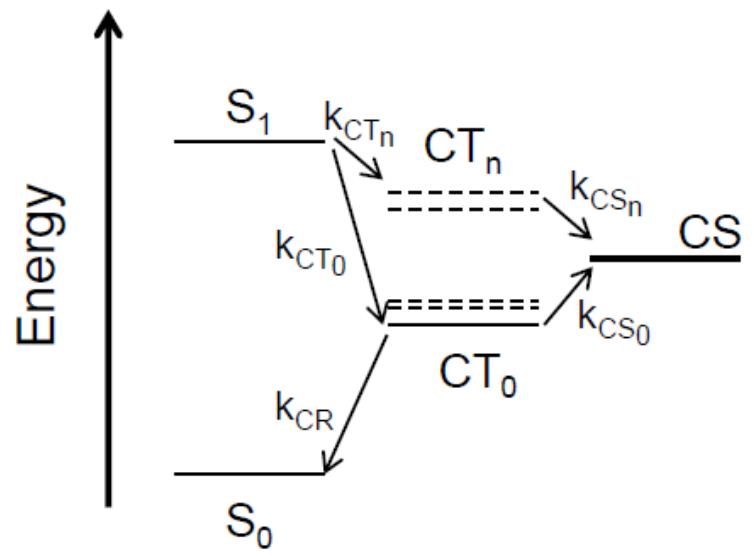

Figure 2. Diagram describing relevant energy levels in the OPV process transforming excitons into free charges. $S_{0}$ represents the singlet ground state of the donor or the acceptor and $S_{1}$, the first singlet excited state formed by light absorption at the bulk heterojunction, $\mathrm{CT}_{\mathrm{n}}$ represents the charge transfer complexes that can be formed upon transfer of an electron (hole) from the donor (acceptor) to the acceptor (donor) and CS represent the final charge separated state where the charge transfer complex has transformed into free charge carriers, a h+-polaron and $\mathrm{e}^{-}$-polaron in the donor and acceptor regions, respectively. The $\mathrm{k}$ terms represent competing transfer rates between various electronic states. Note that the diagram is a simplified picture, e.g., a variety of $\mathrm{S}_{0}, \mathrm{~S}_{1}$ and $\mathrm{CS}$ states exists in a D/A blend layer due to local variations in the molecular order. 


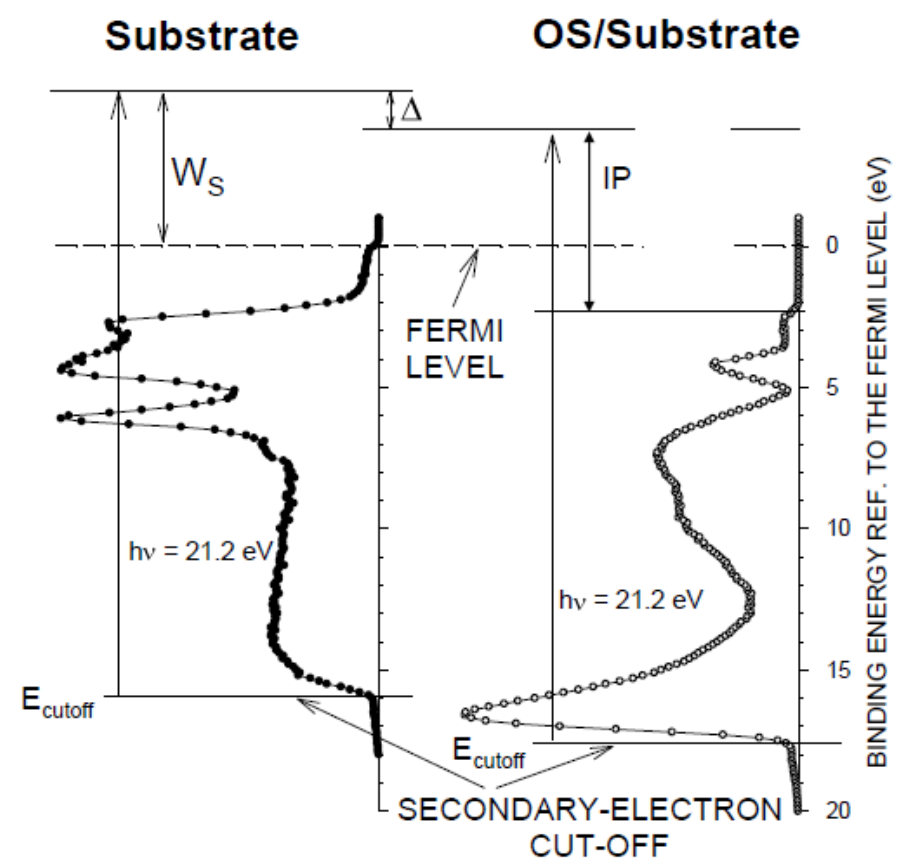

Figure 3. Schematic figure of some of the relevant parameters derived from PES characterization of surfaces and interfaces.

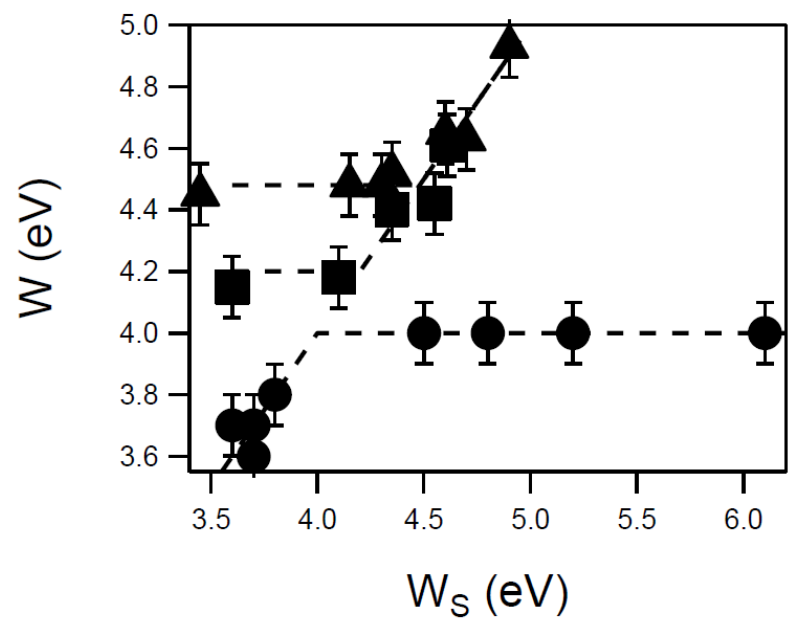

Figure 4. Substrate work function $\left(\mathrm{W}_{\mathrm{S}}\right)$ dependence of the final work function (W) for $\mathrm{C}_{60}$ (triangles), PCBM (squares) and annealed rr-P3HT (circles). 


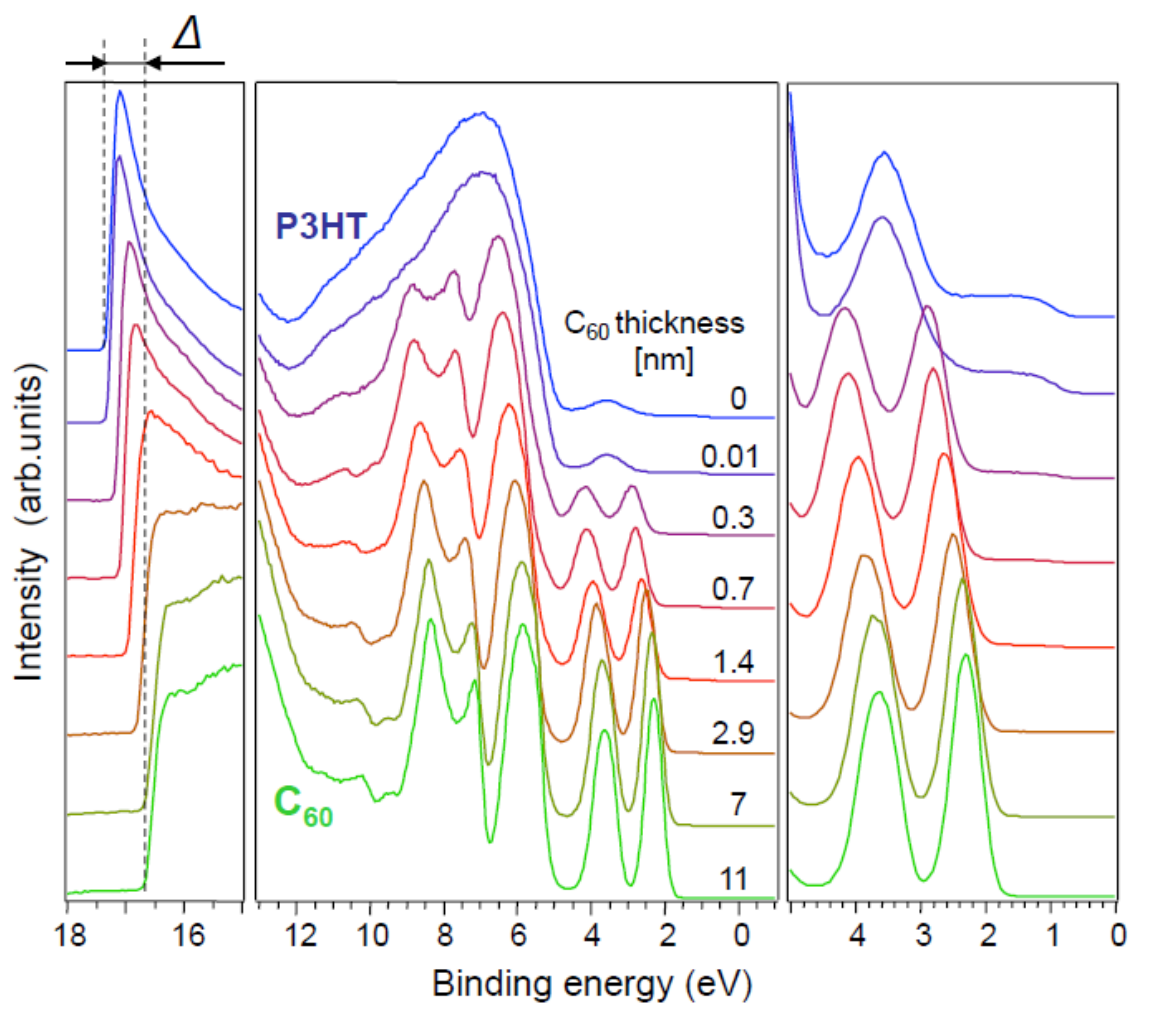

Figure 5. UPS spectra of a $\mathrm{C}_{60} / \mathrm{P} 3 \mathrm{HT}$ sample, recorded during successive steps of $\mathrm{C}_{60}$ deposition. Vacuum level shifts, $\Delta$, were determined from the secondary electron cut-off as shown in the left panel and Eq. 1. The right panel shows a zoom-in of the frontier electronic states, i.e. those closest to the substrate Fermi level situated at $0 \mathrm{eV}$ binding energy.

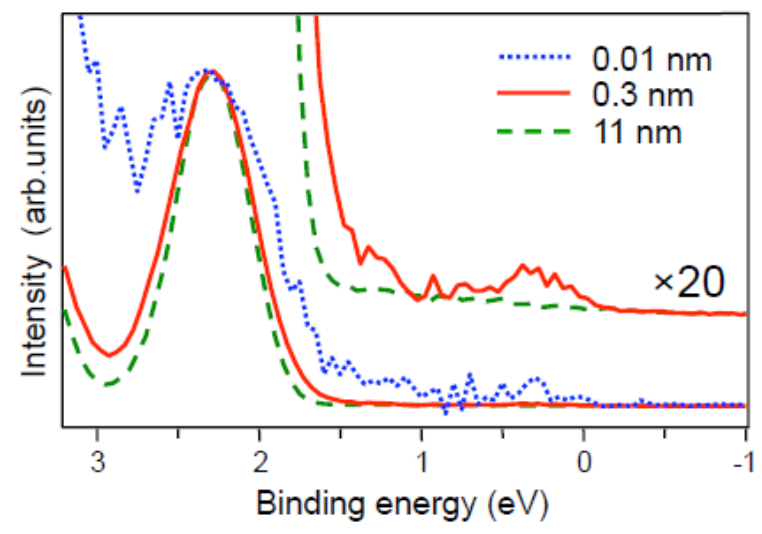

Figure 6. Evidence of the presence of negatively charged $\mathrm{C}_{60}$ molecules is contained in the UPS spectra obtained for the very first stages of $\mathrm{C}_{60} / \mathrm{P} 3 \mathrm{HT}$ interface formation, where spectral weight appears between 1 and $0 \mathrm{eV}$ binding energy. The curves for nominally $0.01 \mathrm{~nm}$ and $0.3 \mathrm{~nm}$ thick $\mathrm{C}_{60}$ overlayers were obtained by subtraction of the P3HT signal from the total UPS intensity. For comparison, a spectrum of an $11 \mathrm{~nm}$ thick $\mathrm{C}_{60}$ film is shown as well. The spectra were scaled and aligned to match the amplitude and position of the $\mathrm{C}_{60} \mathrm{HOMO}$ peak (at about $2.3 \mathrm{eV}$ ) recorded for the thick film. 
Table 1. Results from PES measurements $(\mathrm{hv}=21.2 \mathrm{eV})$ on $\mathrm{rr}-\mathrm{P} 3 \mathrm{HT}$. The positive integer charge transfer energy $\left(\mathrm{E}_{\mathrm{ICT}+}\right)$ and vertical ionization potential (IP), measured for pristine films at RT, at elevated temperatures and after cooling to RT, all done in situ. Experimental error $\pm 0.05 \mathrm{eV}$.

\begin{tabular}{|l|l|l|l|l|l|l|}
\hline rr-P3HT & Pristine sampe, $\mathrm{RT}$ & $50^{\circ} \mathrm{C}$ & $80^{\circ} \mathrm{C}$ & $110^{\circ} \mathrm{C}$ & $150^{\circ} \mathrm{C}$ & $\mathrm{RT}$ \\
\hline $\mathrm{E}_{\mathrm{ICT}+}(\mathrm{eV})$ & 4.40 & 4.40 & 4.35 & 4.25 & 4.05 & 4.00 \\
\hline $\mathrm{IP}(\mathrm{eV})$ & 4.50 & 4.50 & 4.50 & 4.50 & 4.50 & 4.45 \\
\hline
\end{tabular}


Table 2. Lifetime and charge concentration extracted from photoinduced absorption spectroscopy data. Values for the lifetime $(\tau)$ and charge concentration $(n)$ are given for 785 and $514 \mathrm{~nm}$ excitation wavelengths. The values are measured at a $1.25 \mathrm{eV}$ probe energy. The error margins given are estimated from the noise in the data.

\begin{tabular}{|l|c|c|}
\hline & \multicolumn{2}{|c|}{ rr-P3HT } \\
\hline & Pristine & Annealed \\
\hline$\tau_{785}(\mu \mathrm{s})$ & $90 \pm 30$ & $60 \pm 20$ \\
\hline$\tau_{514}(\mu \mathrm{s})$ & $70 \pm 10$ & $35 \pm 5$ \\
\hline $\mathrm{n}_{785}\left(10^{16} \mathrm{~cm}^{-3}\right)$ & $0.46 \pm 0.01$ & $0.90 \pm 0.01$ \\
\hline $\mathrm{n}_{514}\left(10^{16} \mathrm{~cm}^{-3}\right)$ & $14.9 \pm 0.02$ & $25.5 \pm 0.02$ \\
\hline
\end{tabular}


Table 3. Calculated pinning levels $W_{I C T}^{-}$, Eq. 29, for acceptor molecules, and $W_{I C T}^{+}$, Eq. 30, for donor molecules, versus experimental pinning levels.

\begin{tabular}{|l|l|l|l|}
\hline molecule & $W_{I C T}^{-}(\mathrm{eV})$ & $W_{I C T}^{+}(\mathrm{eV})$ & Exp. $(\mathrm{eV})$ \\
\hline $\mathrm{C}_{60}$ & 4.44 & & $4.5[31]$ \\
\hline $\mathrm{PTCDA}(\mathrm{l})$ & 4.74 & & $4.7[60]$ \\
\hline $\mathrm{F}_{16} \mathrm{CuPc}$ & 5.21 & & $5.25[61]$ \\
\hline $\mathrm{CuPc}(\mathrm{s})$ & & 4.41 & $4.35,4.4[62]$ \\
\hline $\mathrm{TTF}$ & & 4.2 & $4.2[63]$ \\
\hline
\end{tabular}

